\title{
Fístula arteriovenosa iliaca-rectal como causa de rectorragia recurrente. Reporte de caso y breve revisión anatómica
}

\author{
Recto-illiac arterio-venous fistula as the cause of recurrent rectal bleeding. \\ Case report and brief anatomical review
}

Alfredo Alonso Sánchez-Betancourt,* Pablo Sibaja-Álvarez**

Palabras clave: Enfermedad rectal, malformación, malformación arteriovenosa.

Key words: Rectal diseases, malformation, arteriovenous malformation.

* Profesor adjunto de Anatomía.

** Catedrático de Cirugía.

Universidad San Judas Tadeo, Costa Rica.

Recibido: 16/05/2016 Aceptado: 21/09/2017

\section{RESUMEN}

La rectorragia copiosa es un reto diagnóstico y terapéutico para los equipos quirúrgicos. Dentro de las causas de sangrado digestivo bajo encontramos que la más frecuente es la enfermedad diverticular, pero puede deberse a una gran cantidad de entidades patológicas. En este artículo se describe un caso de un paciente de 31 años que tuvo un sangrado digestivo bajo masivo y recurrente posterior a una embolización endoarterial a causa de una fístula arteriovenosa bilateral detectada que comunicaba las ramas pélvicas de la arteria iliaca interna con el plexo venoso rectal externo. El procedimiento resultó ser tanto diagnóstico como curativo.

\section{ABSTRACT}

Copious rectal bleeding poses a diagnostic and therapeutic challenge in the surgical wards. The main cause of lower GI bleeding is diverticular disease, but, this condition may be caused by a large number of pathological entities. With regards to rectal bleeding, the main cause of this condition is hemorrhoidal disease. We describe in this article, the case of a 31 year old male, whom after being subjected to an endoarterial embolization, was found to have an arteriovenous fistula communicating the pelvic branches of the interior iliac artery and the external rectal venous plexus. This procedure proved to be both diagnostic and therapeutic for this condition.

\section{INTRODUCCIÓN}

E I sangrado digestivo bajo masivo representa - un reto diagnóstico y terapéutico. Se estima que la incidencia anual de sangrado digestivo bajo es de 20.5 pacientes $/ 100,000,{ }^{1}$ siendo más frecuente en hombres y presenta un importante incremento de incidencia a partir de los 65 años de edad. La principal causa de sangrado digestivo bajo es la enfermedad diverticular, ${ }^{2}$ seguida de la angiodisplasia y el cáncer colorrectal. ${ }^{3}$ En el caso del sangrado rectal y la hematoquecia ${ }^{4}$ en el adulto se observa que la enfermedad hemorroidal es la principal entidad patogénica.

Aproximadamente una de cada cuatro personas tiene un sangrado rectal durante su vida, aunque solamente $41 \%$ de los pacientes que tuvieron un evento de esta índole consultó por esta situación. ${ }^{5}$ En aquellos pacientes que buscan atención médica, el manejo diagnós- tico frente a este fenómeno no es del todo apropiado, ya que frecuentemente se omite la realización de un examen rectal, abdominal y el envío de pruebas complementarias. ${ }^{6}$

La iatrogenia es un importante factor causal de sangrado masivo rectal, ${ }^{7-10}$ igualmente hay múltiples entidades patológicas asociadas de forma infrecuente a rectorragia masiva tales como várices hemorroidales, ${ }^{11}$ hemangiomas, ${ }^{12}$ úlceras rectales asociadas a enfermedades autoinmunes ${ }^{13}$ e infecciones. ${ }^{14,15}$ Posterior a una revisión de las bases de datos de Ebscohost y Google scholar no fue posible documentar en la literatura un caso más de malformación en estos vasos particulares, aunque sí se detectaron otras malformaciones vasculares a nivel de la fosa isquioanal, ${ }^{16}$ las cuales eran bilaterales.

El propósito de este artículo es informar sobre una fístula arteriovenosa entre el plexo 
rectal externo y las ramas de las arterias iliacas internas como la causa de sangrado rectal recidivante y describir brevemente la anatomía vascular del recto, con la finalidad de que nuestra experiencia sea de utilidad en el momento de enfrentarse a un paciente con una presentación clínica similar.

\section{CONSIDERACIONES ANATÓMICAS DE LA VASCULATURA DEL RECTO}

La anatomía vascular del recto es compleja y proviene de varias fuentes. ${ }^{17}$ De acuerdo con Shafik, las dos ramas principales que irrigan el recto son las arterias rectales superiores y las arterias rectales inferiores, ${ }^{18}$ lo que difiere de la concepción tradicional emitida por Drummond, en la cual la principal fuente vascular es la arterial rectal superior. ${ }^{19}$ Según Didio, las arterias rectales medias tienen una presentación variable y se encuentran entre 12 y $56.7 \%$ de los pacientes ${ }^{20,21}$ (Cuadro I).

La arteria rectal superior es la principal fuente de irrigación del recto, ${ }^{21}$ esta arteria da origen a cuatro ramas terminales: rectal transversa, rectal descendente, rectosigmoidea y terminal. ${ }^{17} \mathrm{La}$ arteria rectal inferior irriga el canal anal inferior al músculo elevador del ano. ${ }^{21}$ La arteria rectal media irriga de forma accesoria parte de la capa muscular del recto, en especial, la porción media. ${ }^{22}$ Se recomienda cambiar el término de arterias rectales medias por arterias rectales inferiores y pasar a denominar las actuales arterias rectales inferiores como arterias anales, ya que estas últimas irrigan principalmente el canal anal.

Cuadro I. Variabilidad de la existencia de la arteria rectal media.

Presencia de la arteria rectal media y su distribución pélvica según el autor

Presente (\%) Bilateral (\%) Unilateral (\%)

\begin{tabular}{llll}
\hline Ayoub $^{21}$ & 12 & 40 & 60 \\
Bilhim $^{22}$ & 35.90 & 33 & 66 \\
DiDio $^{20}$ & 56.70 & 36.70 & 63.30 \\
Dujovnyy $^{25}$ & 32 & ------- & ----- \\
Sato $^{23}$ & 34.90 & 20 & 80 \\
Shafik $^{17}$ & 50 & ------- & ----
\end{tabular}

En el caso de las venas rectales medias, éstas también son inconstantes, se presentan en $32 \%$ de las personas, siendo más frecuentes en hombres que en mujeres. ${ }^{23} \mathrm{El}$ drenaje venoso del recto se recibe por medio de dos plexos venosos, el plexo rectal interno o hemorroidal y el plexo rectal externo, formado por la confluencia de las venas rectales superiores, medias e inferiores. ${ }^{24}$ Las venas rectales superiores drenan el recto y el canal anal por arriba de la línea dentada y las otras dos venas drenan el tejido inferior a esta estructura. Las venas rectales superiores continúan en forma de la vena mesentérica inferior. Las venas rectales medias e inferiores van a la vena pudenda y de ahí a la vena iliaca interna. ${ }^{25}$

\section{REPORTE DE CASO}

Se describe el caso de un paciente de 31 años de edad, portador crónico de diabetes insípida central con fístulas perianales intervenidas quirúrgicamente en cuatro ocasiones asociadas a múltiples transfusiones de hemocomponentes, quien consulta al servicio de urgencias por una rectorragia intermitente de dos meses de evolución.

Durante la evaluación inicial se observa que el paciente padece de anemia microcítica hipocrómica, al igual que la presencia de una fístula perianal. Se determina que el paciente requiere una exploración quirúrgica de la fístula.

Es sometido a una exploración anal, la cual arroja la presencia de un granuloma, se realiza curetaje y se obtiene hemostasia durante el procedimiento.

En el día postoperatorio número 1 el paciente mostró nuevamente un cuadro de rectorragia y dolor abdominal intenso, por lo que fue reintervenido. Previo a esta intervención el paciente tenía un nivel de hemoglobina de $8.2 \mathrm{mg} / \mathrm{dl}$.

El sangrado persistió, por lo cual se practicó una tercera intervención en la que se detectó un sangrado por capas en el sitio donde se realizó el curetaje, por tal motivo se efectuó nuevamente hemostasia quirúrgica.

El paciente continuó con cuadros de rectorragia cuantiosa intermitente por los próximos siete días y requirió esquemas agresivos de resucitación con plasma, glóbulos rojos empacados 
y cristaloides, debido a que se tornó hipotenso y a que la anemia microcítica persistía a pesar de las múltiples transfusiones, fluctuando en hemoglobinas que iban de 6.5 a $10 \mathrm{mg} / \mathrm{dl}$. El paciente fue valorado por hematología y no se logró determinar una causa hematológica como responsable del sangrado recidivante.

Se decidió practicar una embolización endoarterial como estrategia para obtener hemostasia. Durante el procedimiento se observó la presencia de una fístula arteriovenosa bilateral del plexo rectal y de las ramas de las arterias iliacas internas, siendo la malformación arteriovenosa derecha la causante del sangrado (Figura 1 y 2).

Se practicó embolización bilateral con la colocación de microcoils y micropartículas con apropiada hemostasia (Figura 3). Posteriormente el paciente permaneció libre de rectorragia y fue egresado siete días posteriores a la embolización.

\section{DISCUSIÓN}

El recto es un órgano altamente vascularizado que recibe irrigación por parte de tres venas, de las cuales una es inconstante. La anatomía vascular del recto es muy variable, hay presencia de venas rectales medias en aproximadamente un tercio de las personas. Al parecer la arteria rectal media es más frecuente que la vena homónima, aunque cuando está presente corre de forma paralela a la arteria. Debido a esta rica fuente vascular el órgano es propenso a sangrados copiosos bajo ciertas circunstancias tales como infecciones, patología vascular, enfermedad autoinmune e iatrogenia.

Se consideró que la causa original del sangrado era una fístula perianal. Más tarde en la evolución del cuadro, después de que múltiples intervenciones no lograron detener la hemorragia, se inició la búsqueda de una causa secundaria del sangrado. En este caso, el paciente mostraba una malformación vascular como la causa del sangrado masivo recidivante, por lo cual la terapia convencional no fue efectiva. Instamos a los médicos clínicos a tomar en cuenta causas poco frecuentes en caso de enfrentarse a un paciente en quien hay fallo terapéutico con las medias curativas típicas.

Los sangrados rectales recidivantes representan un reto particular para el equipo quirúrgico, aunque entre las causas más frecuentes frente a un sangrado a repetición posterior a una intervención quirúrgica apropiada debe valorarse la presencia de una causa secundaria del sangrado, por lo que una valoración multidisciplinaria es importante. Las fístulas
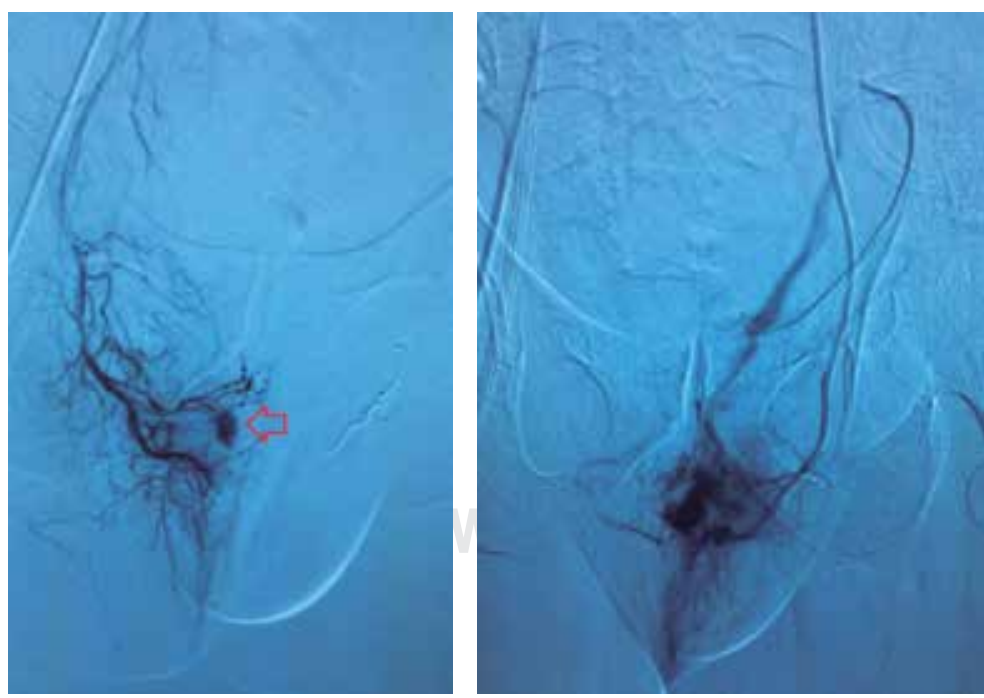

Figura 1 y 2. Malformación arteriovenosa vista por medio de angiografía, nótese la extravasación del medio de contraste a nivel de la anomalía y la fuga al sistema venoso por medio de la fístula arteriovenosa.

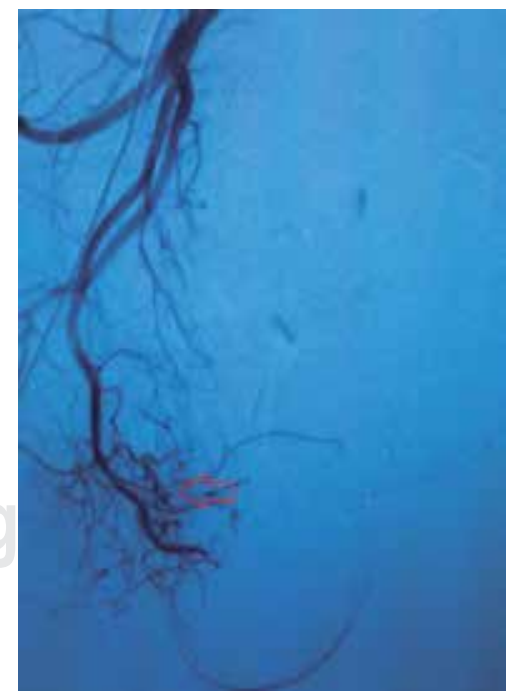

Figura 3. Embolización de la fístula arteriovenosa con colocación de microcoil vista por medio de una arteriografía, nótese la presencia bilateral de coils. 
arteriovenosas son causas poco frecuentes de sangrado rectal, pero deben considerarse dentro del diagnóstico diferencial en casos de sangrado de difícil manejo.

\section{REFERENCIAS}

1. Longstreth GF. Epidemiology and outcome of patients hospitalized with acute lower gastrointestinal hemorrhage: a population-based study. Am J Gastroenterol [Internet]. 1997; 92: 419-424. Available from: http://www.ncbi.nlm.nih.gov/ pubmed/9068461

2. Strate LL. Lower GI Bleeding: Epidemiology and diagnosis. Gastroenterol Clin North Am [Internet]. 2005; 34: 643-664. Available from: http://www.ncbi. nlm.nih.gov/pubmed/16303575

3. Kontopodis N, Spiridakis K, Grigoraki M, Panagiotakis G, Papadakis T, Kokkinakis T, et al. Colon bleeding from both arteriovenous malformation and inflammatory bowel disease. Case report. G Chir [Internet]. 2011; 32: 417-420. Available from: http://www.ncbi.nlm. nih.gov/pubmed/22018216

4. Gralnek IM, Ron-Tal Fisher O, Holub JL, Eisen GM The role of colonoscopy in evaluating hematochezia: a population-based study in a large consortium of endoscopy practices. Gastrointest Endosc [Internet]. 2013; 77: 410-418. Available from: http://www.ncbi. nlm.nih.gov/pubmed/23294756

5. Crosland A, Jones R. Rectal bleeding: prevalence and consultation behaviour. BMJ [Internet]. 1995; 311. Available from: http://www.bmj.com/content/311/70 03/486? linkType $=$ FULL\&resid $=311 / 7003 / 486 \&$ jour nalCode $=$ bmj

6. Weingart SN, Stoffel EM, Chung DC, Sequist TD, Lederman RI, Pelletier SR, et al. Working up rectal bleeding in adult primary care practices. J Eval Clin Pract [Internet]. 2017; 23: 279-287. Available from: http://doi.wiley.com/10.1111/jep.12596

7. Dahshan A. Serious rectal bleeding complicating suction rectal biopsy in a child. W V Med J [Internet]; 110: 34-35. Available from: http://www.ncbi.nlm.nih. gov/pubmed/24902467

8. De Beule T, Carels K, Tejpar S, Van Cleynenbreugel B, Oyen R, Maleux G. Prostatic biopsy-related rectal bleeding refractory to medical and endoscopic therapy definitively managed by catheter-directed embolotherapy: a case report. J Med Case Rep [Internet]. BioMed Central; 2015; 9: 242. Available from: http://www.ncbi.nlm.nih.gov/ pubmed/265113324

9. Beuran M, Stoica B, Tănase I, Negoi I, Păun S. Massive intraoperative bleeding after laparoscopic assisted abdominoperineal resection: a case report and systematic review of the literature. Chirurgia (Bucur) [Internet]; 110: 165-170. Available from: http://www. ncbi.nlm.nih.gov/pubmed/26011840

10. Popek S, Senagore A. Indwelling rectal tubes: an unusual cause of significant rectal bleeding in two critically ill patients. Am Surg [Internet]. 2013; 79: 219-220. Available from: http://www.ncbi.nlm.nih. gov/pubmed/23336667
11. Johansen K, Bardin J, Orloff MJ. Massive bleeding from hemorrhoidal varices in portal hypertension. JAMA J Am Med Assoc [Internet]. American Medical Association. 1980; 244: 2084. Available from: http:// jama.jamanetwork.com/article.aspx?doi $=10.1001 /$ jama.1980.03310180050035

12. Sylla P, Deutsch G, Luo J, Recavarren C, Kim S, Heimann TM, et al. Cavernous, arteriovenous, and mixed hemangioma-lymphangioma of the rectosigmoid: rare causes of rectal bleeding-case series and review of the literature. Int J Colorectal Dis [Internet]. 2008; 23: 653-658. Available from: http:// www.ncbi.nlm.nih.gov/pubmed/18330577

13. Bes C, Dağlı Ü, Yılmaz F, Soy M. Massive lower gastrointestinal bleeding associated with solitary rectal ulcer in a patient with Behçet's disease. Reumatismo [Internet]. 2015; 67: 82-84. Available from: http:// www.ncbi.nlm.nih.gov/pubmed/26492967

14. Álvarez M, Gómez-Polo JC, Lerma JL, Lozano Mde L. Rectal bleeding and microabscesses in a not immunosuppressed patient]. Rev Esp Quimioter [Internet]. 2015; 28: 54-55. Available from: http:// www.ncbi.nlm.nih.gov/pubmed/25690146

15. Azghari I, Bargach A, Moatassim Billah N, Essaoudi MA, Jahid A, Kabbaj N. Ileocecal resection for massive rectal bleeding due to Yersinia enterocolitica: a case report and review of the literature. J Med Case Rep [Internet]. 2016; 10: 6. Available from: http://www. ncbi.nlm.nih.gov/pubmed/26781434

16. Smith WG, Beahrs OH, McDonald JR,. Congenital arteriovenous fistula involving both ischioanal fossae; report of case. Ann Surg [Internet]. 1957; 145: 115118. Available from: http://www.ncbi.nlm.nih.gov/ pubmed/13395291

17. Shafik A, Mostafa H. Study of the arterial pattern of the rectum and its clinical application. Acta Anat (Basel) [Internet]. 1996; 157: 80-86. Available from: http:// www.ncbi.nlm.nih.gov/pubmed/9096745

18. Foster ME, Lancaster JF, Leaper DJ. Functional aspects of ano-rectal vascularity. Acta Anat (Basel) [Internet]. 1985; 123: 30-33. Available from: http://www.ncbi. nlm.nih.gov/pubmed/4050305

19. Drummond $\mathrm{H}$. The arterial supply of the rectum and pelvic colon. Br J Surg [Internet]. 1913; 1: 677685. Available from: http://doi.wiley.com/10.1002/ bjs. 1800010417

20. DiDio LJ, Diaz-Franco C, Schemainda R, Bezerra AJ. Morphology of the middle rectal arteries. A study of 30 cadaveric dissections. Surg Radiol Anat [Internet]. 1986; 8: 229-236. Available from: http://www.ncbi. nlm.nih.gov/pubmed/3107146

21. Ayoub SF. Arterial supply to the human rectum. Acta Anat (Basel) [Internet]. 1978; 100: 317-327. Available from: http://www.ncbi.nlm.nih.gov/ pubmed/619506

22. Bilhim T, Pereira JA, Tinto HR, Fernandes L, Duarte M, O'Neill JE, et al. Middle rectal artery: Myth or reality? Retrospective study with $\mathrm{CT}$ angiography and digital subtraction angiography. Surg Radiol Anat [Internet]. 2013; 35: 517-522. Available from: http:// link.springer.com/10.1007/s00276-012-1068-y

23. Sato K, Sato T. The vascular and neuronal composition of the lateral ligament of the rectum and the rectosacral 
fascia. Surg Radiol Anat. 1991; 13: 17-22. Available from: http://www.ncbi.nlm.nih.gov/pubmed/2053040

24. Caggiati A, Bergan JJ, Gloviczki P, Eklof B, Allegra C, Partsch $\mathrm{H}$, et al. Nomenclature of the veins of the lower limb: Extensions, refinements, and clinical application. J Vasc Surg [Internet]. Churchill Livingstone Inc, New York; 2005; 41: 719-724. Available from: http://linkinghub.elsevier.com/retrieve/pii/ S0741521405001217
25. Dujovny N, Quiros RM, Saclarides TJ. Anorectal anatomy and embryology. Surg Oncol Clin N Am. 2004; 13: 277-293.

Correspondencia:

Dr. Alfredo Alonso Sánchez-Betancourt Tel: (506) 83371547

E-mail: drsanchez@usanjudas.ac.cr 